\title{
Laser Ablation of a Large Tongue Hemangioma with Remifentanil Analgosedation in the ORL Endoscopy Suite
}

\author{
Joshua H. Atkins ${ }^{a, b}$ Jeff E. Mandel ${ }^{a}$ Natasha Mirza ${ }^{b}$ \\ Departments of a Anesthesiology and Critical Care and ${ }^{b}$ Otorhinolaryngology - Head and Neck Surgery, \\ University of Pennsylvania School of Medicine, Philadelphia, Pa., USA
}

\section{Key Words}

Analgosedation - Endoscopy suite - Laser airway

surgery · Opioids • Remifentanil • Respiratory inductance plethysmography

\begin{abstract}
We present a unique, practical, and safe approach to the clinical management of a young male with a large tongue hemangioma who presented for serial surgical treatment of the lesion. Laser ablation was undertaken in the operating room under topical anesthesia with remifentanil analgosedation without the use of supplemental oxygen. Significant involution of the hemangioma was achieved without complication while the patient was awake, cooperative, and able to protect his airway. The application of remifentanil infusion for analgosedation during airway surgery is described. The utility of pharmacokinetic modeling in these applications is discussed along with the use of non-invasive respiratory inductance plethysmography to monitor ventilation during opioid sedation. The concept of analgosedation for airway surgery is introduced and relative risk versus benefit considerations of the approach in comparison to general anesthesia are discussed. This approach can be conceived of as an ORL endoscopy suite model for limited airway procedures.
\end{abstract}

Copyright $\odot 2011$ S. Karger AG, Basel

\section{Introduction}

Endoscopic treatment of laryngotracheal conditions can be performed under general anesthesia in the operating room or by employing local anesthesia in the office. Local anesthesia alone is often insufficient to calm patient anxiety and provide adequate analgesia. Although frequently advocated as the safest approach, general anesthesia is not without associated risks and morbidity. These include systemic reactions to anesthetic drugs, aspiration, hypoxia due to a 'cannot intubate/cannot ventilate scenario', or intubation-related laryngeal trauma that can include bleeding, vocal cord injury, edema, and dental injury. These risks may be amplified in the patient who presents for serial procedures. Analgosedation offers the opportunity for high patient satisfaction and a decreased potential for morbidity from general anesthesia and airway manipulation. Experience with the rapid-onset ultra-short-acting opioid remifentanil has demonstrated utility for a wide range of surgical procedures and exhibited a reasonable safety profile when used appropriately. We present a unique case that demonstrates the utility and safety of remifentanil analgosedation for moderately invasive airway surgery. 

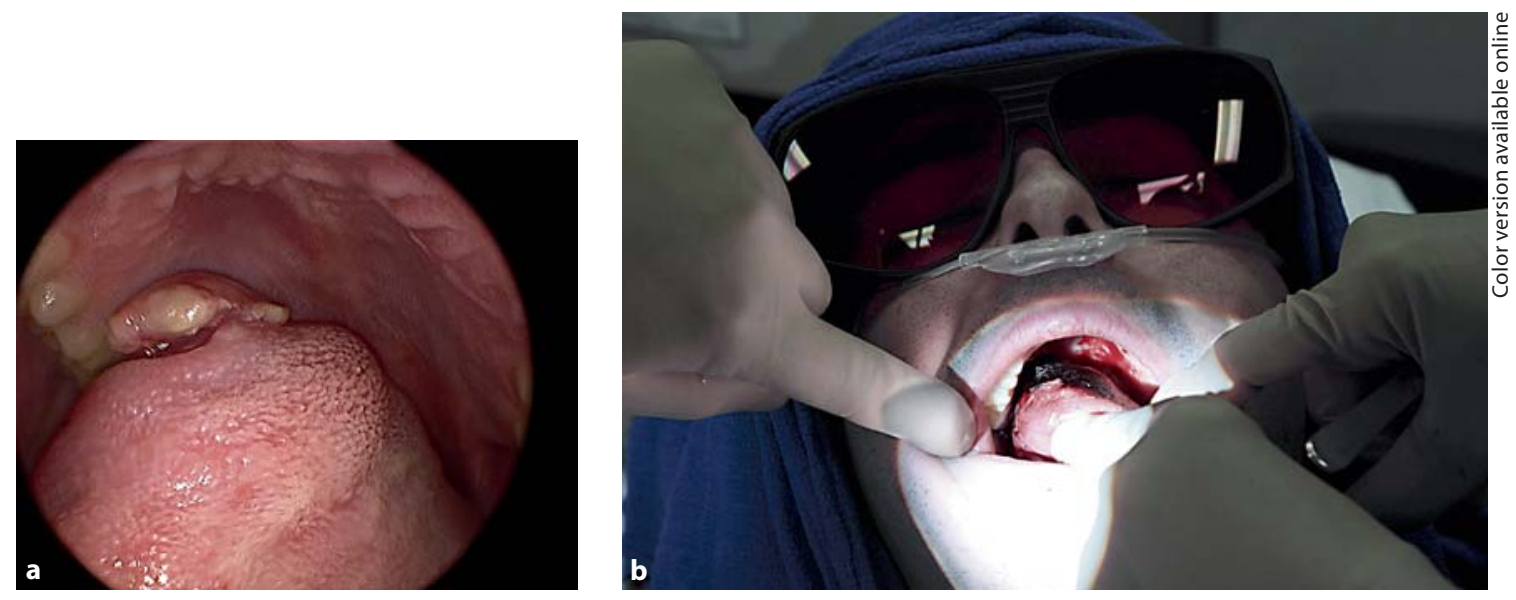

Fig. 1. Tongue lesion prior (a) and subsequent (b) to ablation.

\section{Case Presentation}

The patient is a 22-year-old male $(172.7 \mathrm{~cm}, 65.8 \mathrm{~kg}$, BMI 22) who presented with a bleeding mass of the right posterior tongue. Examination of the mass several years prior at another facility revealed a hemangioma. Recently, the mass increased in size and he experienced multiple episodes of bleeding. Each episode consisted of the loss of 2-3 spoonfuls of bright red blood after which bleeding would spontaneously stop. He denied any respiratory symptoms, swallowing problems, or voice abnormality, but had minor issues with clarity in word articulation. His past medical history was only significant for migraines, and he denied tobacco or significant alcohol use. He is a college student who works parttime as a pharmacy technician.

The head and neck examination was unremarkable apart from a $3 \times 3 \mathrm{~cm}$ bluish mass on the right side of the tongue that extended across the midline and to the right floor of the mouth (fig. 1a). The surface of the mass revealed a scab and a small ulcer. On flexible laryngoscopy, the bluish mass extended to the base of the tongue and the vallecula on the right side as well. An MRI showed that the mass extended into the pterygoid space and involved more than half of the tongue.

The patient was taken to the operating room for a laser ablation of the hemangioma. Due to the large size of the lesion and involvement of the tongue and tongue base, intubation was considered very risky as it could potentially traumatize the hemangioma and cause significant bleeding. The patient gave consent for a possible tracheostomy and general anesthesia. However, the surgical preference and plan was to proceed with the surgery under topical anesthesia with anesthesiologist-administered analgosedation. The patient was amenable to the planned approach.

In the holding area, topical anesthesia of the airway was obtained with nebulized lidocaine $(2 \%, 8 \mathrm{ml}, 6 \mathrm{l} / \mathrm{min}, 10 \mathrm{~min}) . \mathrm{He}$ was brought to the operating room and standard monitors were applied. Supplemental topical anesthesia was accomplished with $5 \%$ lidocaine lollipop and atomized $2 \%$ lidocaine delivered to the posterior pharynx. The patient was separately enrolled in an IRB- approved research study of the effects of remifentanil on respiratory parameters. He was fitted with size 32 respiratory inductance plethysmography bands on the thorax and abdomen that were attached to the Inductotrace oscillator system (Ambulatory Monitoring Systems, Ardsley, N.Y., USA). These bands provide noninvasive continuous measurement of minute ventilation. For the induction and emergence periods, a tight-fitting face mask attached to a pneumotachograph was applied for calibration of flow to the output signal of the Inductotrace. Estimated effect-site concentration of remifentanil based on the Minto pharmacokinetic model was simulated in real time [1].

The procedure was performed in the seated position $\left(\right.$ at $70^{\circ}$ ). Intravenous ondansetron (4 mg), dexamethasone $(10 \mathrm{mg})$, and cefazolin $(1,000 \mathrm{mg})$ were administered. Analgosedation was initiated with remifentanil $(50 \mu \mathrm{g} / \mathrm{ml})$ delivered by rapid bolus $(0.25$ $\mu \mathrm{g} / \mathrm{kg}$ ) through a 20 -gauge intravenous catheter in the left hand with an Alaris infusion pump. A continuous infusion was started at $0.05 \mu \mathrm{g} / \mathrm{kg} / \mathrm{min}$. Depth of analgosedation was increased stepwise $(0.25 \mu \mathrm{g} / \mathrm{kg}$ bolus; $0.02-0.05 \mu \mathrm{g} / \mathrm{kg} / \mathrm{min}$ steps $)$ until a respiratory rate $<10$ was noted. Moderate desaturation occurred while breathing room air that was promptly remedied by instructing the patient to take several deep breaths. The mask was removed and replaced with a $\mathrm{CO}_{2}$-sensing nasal cannula delivering $3 \mathrm{l} / \mathrm{min}$ of oxygen that was turned off at the start of the procedure due to planned laser use. The patient remained entirely responsive to verbal commands throughout the procedure. Additional remifentanil was administered in the doses described as deemed clinically indicated for patient comfort.

After adequate laser protection of the patient and the OR staff, the KTP laser fiber was used to ablate the surface of the hemangioma. Brisk surface bleeding was controlled with a suction Bovie. Intralesional ablation was performed at a higher wattage, and the patient was asked to close and rest his mouth between each session of laser use. To address the posterior aspect of the hemangioma, the patient was asked to protrude his tongue and the laser fiber was passed through a curved 17-gauge hollow needle and the ablation was effectively performed. An intralesional dexametha- 
Fig. 2. Respiratory parameters and modeled remifentanil effect-site concentration.

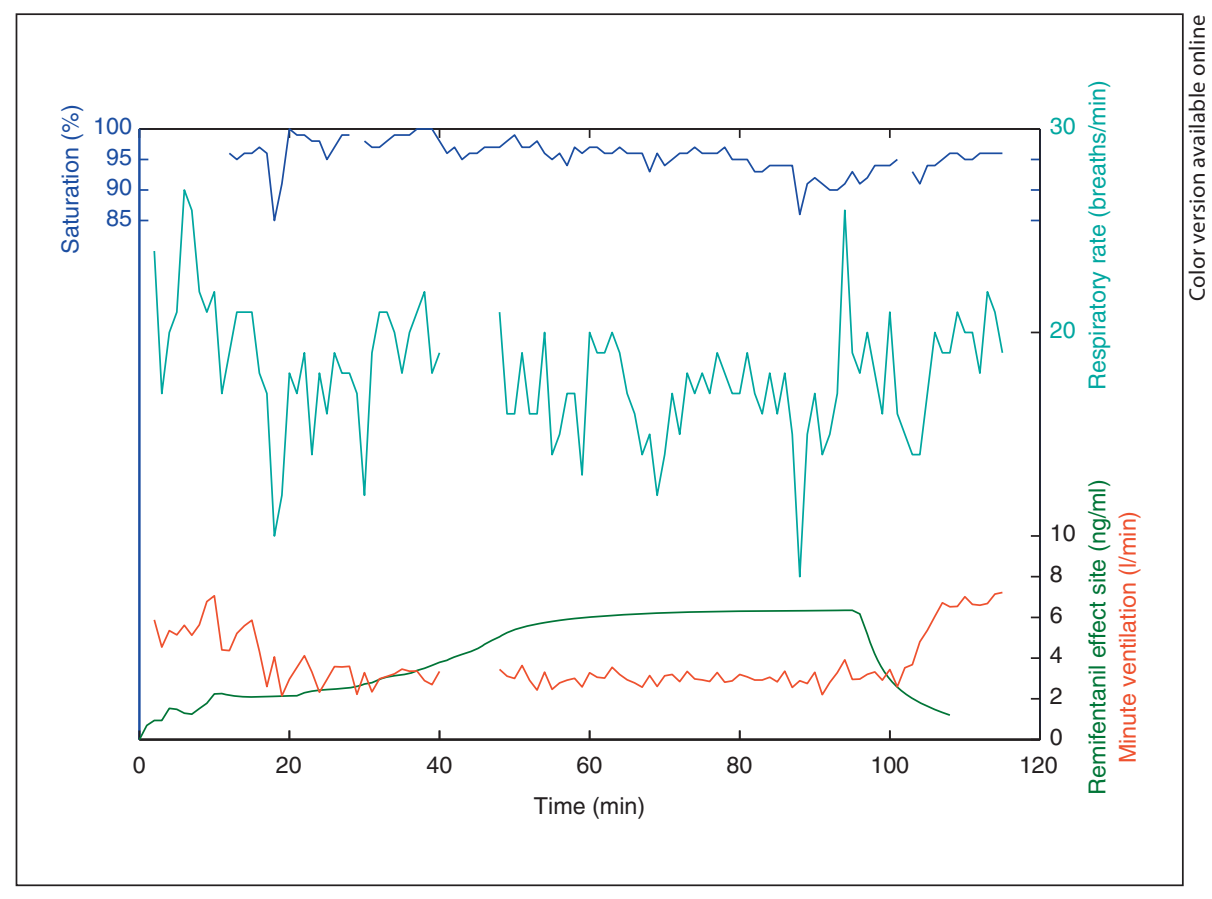

sone injection was performed with surface coagulation of the sites where the needle was introduced as necessary to control the oozing. The procedure lasted about $80 \mathrm{~min}$. Approximately $30 \%$ involution of the hemangioma was achieved with the patient awake, able to effectively protect his airway, and follow commands (fig. 1b). This included self-protrusion of the tongue upon command in order to maximize surgical exposure as needed.

No decrease in dose was needed to avoid oversedation during the procedure. Supplemental oxygen was not used during the laser resection. Upon completion of the procedure, the remifentanil infusion was stopped and the mask/pneumotachograph apparatus used during induction was reapplied for repeat flow calibration. Figure 2 depicts the respiratory trend of parameters measured during the case. The nadir oxygen saturation was $83 \%$, which occurred transiently during induction on room air. Otherwise saturation was maintained at $100 \%$ with supplemental oxygen at $3 \mathrm{l} / \mathrm{min}$ or $>88 \%$ on room air throughout the case. Minute ventilation on initiation of remifentanil was approximately $6 \mathrm{l} /$ $\mathrm{min}$. This decreased after remifentanil loading to a mean value of approximately $3.5 \mathrm{l} / \mathrm{min}$ during the steady-state maintenance phase (as measured by non-invasive respiratory inductance plethysmography) and then rapidly increased after remifentanil cessation to $7 \mathrm{l} / \mathrm{min}$. The peak remifentanil infusion was $0.28 \mu \mathrm{g} / \mathrm{kg} /$ min during the height of the resection. The total remifentanil dose was $1,153 \mu \mathrm{g}$ by infusion and $146 \mu \mathrm{g}(2.2 \mu \mathrm{g} / \mathrm{kg})$ by bolus. The peak simulated effect-site concentration of remifentanil was approximately $6 \mathrm{ng} / \mathrm{ml}$, as shown in figure 2 .

On arrival in the post-anesthesia care unit, the patient was alert, oxygen saturation was $98 \%$ on room air, respirations were 15 breaths/min, blood pressure 143/65, heart rate 70 beats/min, and Aldrete Score was 10. He was given $0.2 \mathrm{mg}$ IV hydromorphone for a pain score of 5 , and later $50 \mu \mathrm{g}$ of fentanyl for a head- ache with a pain score of $3 / 10$ and $12.5 \mathrm{mg}$ of intravenous promethazine for mild nausea.

He was admitted for overnight airway observation and did extremely well. He was able to start an oral intake within $4 \mathrm{~h}$ and was discharged home with minimal pain on oral steroids and antibiotics. He was satisfied with his perioperative experience.

\section{Discussion}

Remifentanil has been sporadically described in the literature for analgosedation with no reports of use for moderately invasive airway surgery where relative control over respiratory parameters is critical [2]. Remifentanil is a synthetic opioid that is metabolized rapidly by non-specific esterases in the plasma to metabolites with clinically insignificant pharmacologic activity. When used alone, the effective half time for effect-site levels to drop by $50 \%$ is approximately $3 \mathrm{~min}$, and does not vary with the duration of the infusion. Remifentanil also offers the advantage of rapid effect-site equilibration (1 min) that allows therapeutic steady-state effect-site concentrations to be achieved in a time frame that is complementary to surgeon needs.

As with all opioids, respiratory depression is the primary side effect of clinical concern. Pharmacokinetic models have been developed that are useful to predict 
remifentanil effect-site concentration [1]. Dose-response curves for remifentanil to produce analgesia to controlled noxious stimuli have been published [3]. Opioids induce respiratory depression primarily by blunting the response of the chemoreceptors to $\mathrm{CO}_{2}$ (i.e. increasing the apneic threshold). An optimal dosing strategy, as evidenced here, reduces the risk of apnea by slow titration to allow the gradual accumulation of carbon dioxide necessary to activate the less sensitive chemoreceptor. Once a new steady state is achieved, with the patient breathing at a reduced minute ventilation, the ongoing risk of apnea is minimal. This was studied in healthy volunteers where it was demonstrated that gradual titration of remifentanil in the awake patient can avoid the apnea associated with the same effect-site concentration arrived at by rapid titration [4]. The findings of this case, in which remifentanil was increased stepwise over 30 min under surgical conditions, are consistent with the model developed in healthy volunteers and should be broadly applicable to analgosedation for airway surgery.

Many patients with airway pathology for ablative procedures will present for sequential debulking or treatment. Modeling of the effect-site concentration required to achieve adequate analgosedation may offer a useful additional parameter to optimize remifentanil dosing for subsequent procedures. Nasal capnometry is one standard for monitoring of respiration during sedation but is frequently unreliable. We employed respiratory inductance plethysmography, a standard modality in sleep laboratories, which can detect respiration and obstruction while allowing quantitative estimation of minute ventilation.

Respiratory depression with associated hypoxemia is a possibility that should be adequately anticipated. Immediate cessation of the infusion and patient stimulation should be attempted. Preparation for resuscitation should also include: (1) equipment for bag-mask ventilation with $100 \%$ oxygen and intubation, and (2) naloxone for rapid pharmacologic reversal.

The ORL endoscopy suite model (i.e. office-based airway procedures in the OR under limited anesthesia) has significant potential advantages to patient and surgeon. Innovative sedation approaches under the direction of an anesthesiologist with the full resources of the operating room can be implemented. Procedures such as the one described here for which a default general anesthesia carries significant risks and disadvantages can be safely performed in a controlled environment. Procedures planned for limited invasiveness that must be converted to general anesthesia can be done immediately. Minimally invasive office-type procedures can be offered to patients

who are reluctant to go without sedation with time and recovery profiles that closely approximate an office visit.

Effective implementation of the model requires appropriate patient selection, surgeon comfort with the concept of awake procedures, patient willingness to forgo reliable amnesia for the benefit of respiratory safety and rapid recovery, and close collaboration with anesthesiologists familiar with the pharmacokinetic/pharmacodynamic profile of remifentanil.

This case highlights the safe and effective implementation of the ORL endoscopy suite model employing remifentanil analgosedation with topicalization.

We emphasize the critical need for cooperation between anesthesiologists and otolaryngologists in the management of challenging airway cases. Topicalized fiberoptic naso- or orotracheal intubation is one alternative that ostensibly decreases the risk of airway soiling and hypoxia. This approach is not without issues. These include the difficulty of nasotracheal intubation with a laser tube, the potential for epistaxis or trauma to the hemangioma during intubation precipitating significant bleeding and airway compromise, the risk of disruption of delicate hemostasis during bucking and coughing at the time of emergence, and the standard risks and recovery profile of general anesthesia. These risks would be incurred during each episode of ablation. In the opinion of the operative surgeon, a chronic tracheostomy would have been the safest alternative to analgosedation. The awake laser procedure allows the surgeon to perform the procedure safely and repeatedly without significantly impacting quality of life with a chronic tracheostomy in a young patient or taking undue risk in repeated airway management.

References

ORL 2011;73:166-169
1 Minto CF, Schnider TW, Shafer SL: Pharmacokinetics and pharmacodynamics of remifentanil II model application. Anesthesiology 1997;86:24-33.

-2 Mesolella M, Lamarca S, Galli V, Ricciardiello F, Cavaliere M, Lengo M: Use of remifentanil for sedo-analgesia in stapedotomy: personal experience. Acta Otorhinolaryngol Ital 2004;24:315-320.

3 Kern SE, Xie G, White JL, Egan TD: A response surface analysis of propofol-remifentanil pharmacodynamic interaction in volunteers. Anesthesiology 2004;100:13731381.

-4 Olofsen E, Boom M, Nieuwenhuijs D, Sarton E, Teppema L, Aarts L, Dahan A: Modeling the non-steady state respiratory effects of remifentanil in awake and propofol-sedated healthy volunteers. Anesthesiology 2010; 112:1382-1395. 